\title{
Response of Four Dry Bean Market Classes to Pre-Emergence Applications of Pyroxasulfone, Sulfentrazone and Pyroxasulfone plus Sulfentrazone
}

\author{
Allison N. Taziar'1, Nader Soltani' ${ }^{1}$ Christy Shropshire1, Darren E. Robinson', \\ Mitch Long' ${ }^{2}$ Chris L. Gillard', Peter H. Sikkema1 \\ ${ }^{1}$ University of Guelph Ridgetown Campus, Ridgetown, Canada \\ ${ }^{2}$ FMC Corporation, Saskatoon, Canada \\ Email: soltanin@uoguelph.ca
}

Received 12 May 2016; accepted 11 June 2016; published 15 June 2016

Copyright (C) 2016 by authors and Scientific Research Publishing Inc.

This work is licensed under the Creative Commons Attribution International License (CC BY).

http://creativecommons.org/licenses/by/4.0/

(c) (†) Open Access

\section{Abstract}

Only one herbicide mode of action (ALS inhibitor) is currently available to Ontario dry bean producers for soil-applied broadleaf weed control. Four field studies were conducted over two years $(2014,2015)$ to examine the tolerance of four market classes of dry beans to sulfentrazone $(210$ and $\left.420 \mathrm{~g} \cdot \mathrm{ai}^{\mathrm{h}} \mathrm{ha}^{-1}\right)$ and pyroxasulfone (100 and $\left.200 \mathrm{~g} \cdot \mathrm{ai} \cdot \mathrm{ha}^{-1}\right)$ applied alone and in combination. The registration of these two herbicides would provide Ontario dry bean producers with two additional modes of action for broadleaf weed control. Pyroxasulfone caused up to $23 \%, 6 \%, 7 \%$ and $10 \%$ injury in adzuki, kidney, small red Mexican and white bean, respectively; sulfentrazone caused up to $51 \%, 12 \%, 15 \%$ and $44 \%$ injury and the combination caused up to $90 \%, 23 \%, 29 \%$ and $62 \%$ injury, respectively. Kidney and small red Mexican bean density, height, seed moisture content and yield were not affected. Pyroxasulfone $\left(200 \mathrm{~g} \cdot \mathrm{ai} \cdot \mathrm{ha}^{-1}\right)+$ sulfentrazone $\left(420 \mathrm{~g} \cdot \mathrm{ai} \cdot \mathrm{ha}^{-1}\right)$ reduced adzuki and white bean density, shoot dry weight, height and yield. This study concludes that pyroxasulfone $\left(100 \mathrm{~g} \cdot \mathrm{ai} \cdot \mathrm{ha}^{-1}\right)+$ sulfentrazone $\left(210 \mathrm{~g} \cdot \mathrm{ai} \cdot \mathrm{ha} \mathrm{a}^{-1}\right)$ applied PRE can be safely used to control weeds in Ontario kidney and small red Mexican bean production.

\section{Keywords}

Adzuki Bean (Erimo), Kidney Bean (Red Hawk), Small Red Mexican Bean (Merlot), White Bean (T9905), Crop Injury, Plant Density, Plant Height, Seed Moisture Content, Tolerance, Yield 


\section{Introduction}

In 2015, Ontario dry bean growers produced 115,000 tonnes of white and coloured dry beans (Phaseolus vulgaris L.) on 52,600 ha [1]. Dry bean market classes commonly grown in Ontario include black, cranberry, kidney, otebo, small red Mexican, white (navy), and adzuki (Vigna angularis (Willd) Ohwi \& Ohashi) bean. One of the most critical aspects of dry bean production is weed control, as weed interference has been reported to cause up to 68\% yield loss [2]-[4]. Currently, Ontario dry bean producers are limited to two acetolactate synthase (ALS) inhibitor herbicides for soil-applied broadleaf weed control, to which there are currently more than ten resistant weed species in the province of Ontario [5]. Therefore, another mode of action is needed for soil-applied broadleaf weed control in order to chemically control these weeds.

Sulfentrazone is a protoporphyrinogen oxidase (PPO) inhibitor herbicide that is recently registered for use in Ontario soybean (Glycine max L.). Sulfentrazone inhibits the PPO enzyme, causing an excess of protoporphyrinogen IX in the chloroplast of the cell [6]. The excess then leaks out of the chloroplast into the cyto-phyrinogen, where various reactions convert it to free oxygen radicals. These radicals disrupt cell membranes, resulting in cellular leakage, loss of cell function, and cell death [7]. Sulfentrazone controls broadleaf weeds such as pigweeds (Amaranthus spp.), common lambsquarters (Chenopodium album L.) and wild buckwheat (Polygonum convolvulus L.), and grasses such as giant foxtail (Setariafaberii Hermm.), fall panicum (Panicum dichotomiflorum Michx.) and witchgrass (Panicum capillare L.) [8]-[11].

Pyroxasulfone is an isoxazoline herbicide that is most effective when applied pre-emergence (PRE) [12] [13]. It is a very-long-chain fatty acid biosynthesis inhibitor that is applied at much lower rates than other group 15 herbicides [14]. Pyroxasulfone primarily controls annual grass species such as barnyard grass (Echinochloa crusgalli (L.) Beauv.), large crabgrass (Digitaria sanguinalis (L.) Scop.), foxtails (Setaria spp.), wild-proso millet (Panicum miliaceum L.) and fall panicum (Panicum dichotomiflorum Michx.), but also controls broadleaf species such as velvetleaf (Abutilon theophrasti Medik.), pigweeds (Amaranthus spp.), common lambs quarters, common ragweed (Ambrosia artemisiifolia L.), and wild mustard (Sinapis arvensis L.) [15] [16]. In Ontario, pyroxasulfone is currently only available in a premix or co-pack with other active ingredients for weed control in maize and soybean crops.

There is little information on dry bean tolerance to sulfentrazone, pyroxasulfone or their combination. The objective of this study was to determine the tolerance of four market classes of dry beans to PRE applications of sulfentrazone, pyroxasulfone, and a combination of the two.

\section{Materials and Methods}

Four field trials were conducted at the University of Guelph Ridgetown Campus, Ridgetown and Huron Research Station, Exeter in Ontario, Canada over two years (2014, 2015). The 2014 Ridgetown site was a loamy soil with sand, silt and clay content of 40\%, 35\% and 25\%, respectively, pH of 6.6 and organic matter content of 7.1\%. The 2014 Exeter site was a Brookston clay loam, with 41\% sand, 40\% silt, 19\% clay, pH of 7.8 and organic matter content of 3.8\%. In 2015, soil at Ridgetown was a sandy clay loam with 53\% sand, 27\% silt, $20 \%$ clay, $\mathrm{pH}$ of 7.0 and organic matter content of 5.3\%. The soil at Exeter in 2015 was a silty clay loam with $18 \%$ sand, $46 \%$ silt, $36 \%$ clay, pH of 7.5 and organic matter content of $4.7 \%$. Seedbeds were prepared by an autumn moldboard ploughing followed by two passes in the spring with a cultivator with rolling basket harrows.

Experiments were arranged in split-plot design, with herbicide as the main plot and bean market class as the sub-plot. Bean market classes included adzuki (“Erimo”), kidney (“Red Hawk”), small red Mexican (“Merlot”), and white (“T9905”) bean. Each experiment had four replicates. Plots in Ridgetown were $6 \mathrm{~m}$ wide by $8 \mathrm{~m}$ long, and consisted of two rows of each market class spaced $75 \mathrm{~cm}$ apart. Plots in Exeter were $3 \mathrm{~m}$ wide by $10 \mathrm{~m}$ long and consisted of 1 row of each market class spaced $75 \mathrm{~cm}$ apart. In Ridgetown, kidney bean was seeded at approximately 175,000 seeds ha ${ }^{-1}$ while adzuki, white and small red Mexican beans were seeded at approximately 235,000 seeds $\mathrm{ha}^{-1}$. In Exeter, kidney and small red Mexican beans were seeded at 200,000 seeds ha ${ }^{-1}$, adzuki bean was seeded at 275,000 ha ${ }^{-1}$, and white bean was seeded at 250,000 seeds ha ${ }^{-1}$. All experiments were seeded 4 to $5 \mathrm{~cm}$ deep between late May and early June. Herbicide treatments (based on manufacturer's recommended rate) included an untreated control, pyroxasulfone at 100 and $200 \mathrm{~g} \cdot \mathrm{ai} \cdot \mathrm{ha}^{-1}$, sulfentrazone at 210 and $420 \mathrm{~g} \cdot \mathrm{ai} \cdot \mathrm{ha}^{-1}$, pyroxasulfone $\left(100 \mathrm{~g} \cdot \mathrm{ai}^{\circ} \mathrm{ha}^{-1}\right)+$ sulfentrazone $\left(210 \mathrm{~g} \cdot \mathrm{ai} \cdot \mathrm{ha}^{-1}\right)$, and pyroxasulfone $\left(200 \mathrm{~g} \cdot \mathrm{ai} \cdot \mathrm{ha}^{-1}\right)+$ sulfentrazone $\left(420 \mathrm{~g} \cdot \mathrm{ai} \cdot \mathrm{ha}^{-1}\right)$. Herbicides were applied with a $\mathrm{CO}_{2}$ pressurized backpack sprayer calibrated to deliver $200 \mathrm{~L} \mathrm{ha}^{-1}$ at $240 \mathrm{kPA}$ using a 1 m handheld boom with three ULD 110-02 nozzles (Hypro, New Brighton, 
MN) spaced $0.5 \mathrm{~m}$ apart. Plots were not irrigated but were fertilized according to recommended Ontario agronomic crop production practices. All plots were maintained weed-free the entire season by hand-hoeing to eliminate the confounding factor of weed interference.

Visible crop injury was assessed at 1, 2, 3, 4, 6 and 8 weeks after crop emergence (WAE). Plant density and shoot dry weight were determined at 3 WAE by counting the number of plants in a metre of row for each market class in each treatment, clipping them at the soil surface, placing them in a separate paper bag, drying in a kiln and weighing once dry. At 6 WAE, plant height was determined by randomly selecting ten plants in each market class in each treatment and averaging their heights. Seed moisture content was determined at maturity. Yield was determined at maturity and adjusted to $15 \%$ moisture for adzuki bean and $18 \%$ moisture for kidney, small red Mexican and white bean.

\section{Statistical Analysis}

Statistical Analysis Software (SAS) v. 9.4 (SAS Institute Inc., NC) was used to perform an analysis of variance on all data. Data were partitioned into the fixed effects of herbicide treatment, bean market class and their interaction, and the random effects of environment (year-location combination), block within environment, and interactions between environment and each of the fixed effects. The significance of the fixed and random effects was determined using the F-test and Z-test, respectively. The UNIVARIATE procedure was used to produce residual plots and Shapiro-Wilk statistics to analyze the homogeneity and normality of the residuals under various transformations. The transformation that produced the highest Shapiro-Wilk statistic was used to analyze each data set. Injury, biomass, and seed moisture data were log transformed, while yield and height data were arcsine square root transformed. The MIXED procedure was used to obtain the least squares means and differences of least squares means to achieve means separation between treatments at a Type 1 error of 0.05 . Orthogonal contrasts were also performed. Data were back transformed for presentation on the original scale.

\section{Results and Discussion}

Statistical analysis determined there was no interaction between bean market class and herbicide treatment for injury at 1 and 2 WAE (Table 1); therefore the main effect of herbicide will be discussed separately. There was an interaction between dry bean market class and herbicide treatment for plant density, shoot dry weight, plant height, seed moisture content and yield (Table 2). Therefore, only the interaction effects will be discussed.

\subsection{Crop Injury}

Crop injury symptoms included leaf malformation, marginal leaf necrosis, stunting, and reduced plant stand. At 1 and 2 WAE, pyroxasulfone (100 and $200 \mathrm{~g} \cdot a \cdot \cdot \mathrm{ha}^{-1}$ ) caused up to 3\% and 7\% injury, respectively (Table 1). Sulfentrazone $\left(210 \mathrm{~g} \cdot \mathrm{ai} \cdot \mathrm{ha}^{-1}\right)$ caused up to $8 \%$ injury and was equivalent to either rate of pyroxasulfone, whilesulfentrazone $\left(420 \mathrm{~g} \cdot a \cdot \mathrm{ha}^{-1}\right)$ caused up to $23 \%$ injury. The combination of pyroxasulfone $\left(100 \mathrm{~g} \cdot \mathrm{ai} \cdot \mathrm{ha}^{-1}\right)$ and sulfentrazone $\left(210 \mathrm{~g} \cdot \mathrm{ai}^{-\mathrm{ha}^{-1}}\right)$ caused up to $11 \%$ injury, which was equivalent to sulfentrazone alone but greater than pyroxasulfone at $2 \mathrm{WAE}$. Similarly, pyroxasulfone $\left(200 \mathrm{~g} \cdot \mathrm{ai} \cdot \mathrm{ha}^{-1}\right)+$ sulfentrazone $\left(420 \mathrm{~g} \cdot \mathrm{ai} \cdot \mathrm{ha}^{-1}\right)$ produced up to $42 \%$ injury which was not different from sulfentrazone $\left(420 \mathrm{~g} \cdot \mathrm{ai} \cdot \mathrm{ha}^{-1}\right)$ alone.

At 3, 4, 6 and 8 WAE, injury was greatest in adzuki bean, followed by white, small red Mexican, then kidney bean (Table 1). Pyroxasulfone (100 g/ai ha ${ }^{-1}$ ) produced the least amount of injury of the herbicide treatments, causing $5 \%$ injury or less in all market classes (Table 3). Pyroxasulfone $\left(200 \mathrm{~g} \cdot \mathrm{ai} \cdot \mathrm{ha}^{-1}\right)$ caused greater injury than the $100 \mathrm{~g} \cdot$ ai $\cdot \mathrm{ha}^{-1}$ rate in adzuki bean at 4 and $6 \mathrm{WAE}$. Pyroxasulfone $\left(200 \mathrm{~g} \cdot \mathrm{ai} \cdot \mathrm{ha}^{-1}\right)$ caused up to $23 \%, 6 \%$, $7 \%$, and $10 \%$ injury in adzuki, kidney, small red Mexican and white bean, respectively. The level of injury observed in adzuki and white bean is consistent with other studies examining pyroxasulfone in dry bean, but the injury observed in kidney and small red Mexican bean were much lower than the $10 \%$ to $21 \%$ injury caused by pyroxasulfone (209 $\mathrm{g} \cdot \mathrm{ai} \cdot \mathrm{ha}^{-1}$ ) in other studies using the same cultivars [17] [18]. Weather conditions (rainfall, temperature, etc.) and soil factors affecting herbicide availability may have contributed to the differences among studies.

Sulfentrazone ( $\left.210 \mathrm{~g} \cdot \mathrm{ai}^{\mathrm{h}} \mathrm{ha}^{-1}\right)$ caused up to $17 \%$ injury in adzuki bean, $4 \%$ injury in kidney and small red Mexican bean, and 16\% injury in white bean (Table 3), which is consistent with a study by Soltani et al. [19]. The high rate of sulfentrazone $\left(420 \mathrm{~g} \cdot \mathrm{ai} \cdot \mathrm{ha}^{-1}\right)$ caused greater injury than the $210 \mathrm{~g} \cdot \mathrm{ai} \cdot \mathrm{ha}^{-1}$ rate in small red 
Table 1. Visible injury of four market classes of dry bean due to pyroxasulfone and sulfentrazone, alone and in combination, applied PRE in 2014 and 2015 at the Huron Research Station, Exeter, ON and University of Guelph Ridgetown Campus, Ridgetown, ON.

\begin{tabular}{|c|c|c|c|c|c|c|}
\hline \multirow{2}{*}{ Main Effects ${ }^{\mathrm{a}}$} & \multicolumn{6}{|c|}{ Dry Bean Injury } \\
\hline & $1 \mathrm{WAE}^{\mathrm{a}}$ & $2 \mathrm{WAE}^{\mathrm{b}}$ & $3 \mathrm{WAE}$ & $4 \mathrm{WAE}$ & $6 \mathrm{WAE}$ & $8 \mathrm{WAE}$ \\
\hline & \multicolumn{6}{|c|}{$\%$} \\
\hline Dry Bean Market Class & $*$ & ** & ${ }^{* *}$ & $* *$ & $* *$ & $*$ \\
\hline Adzuki & $14^{\mathrm{b}}$ & $21^{\mathrm{b}}$ & 27 & 25 & 16 & 11 \\
\hline Kidney & $5^{\mathrm{a}}$ & $6^{\mathrm{a}}$ & 7 & 7 & 3 & 3 \\
\hline Small Red Mexican & $8^{\mathrm{ab}}$ & $7^{\mathrm{a}}$ & 7 & 7 & 4 & 2 \\
\hline White & $15^{\mathrm{b}}$ & $15^{\mathrm{b}}$ & 16 & 14 & 7 & 4 \\
\hline Herbicide Treatment (g·ai $\cdot \mathrm{ha}^{-1}$ ) & $* *$ & $* *$ & $* *$ & $* *$ & $* *$ & $* *$ \\
\hline Untreated & $0^{\mathrm{a}}$ & $0^{\mathrm{a}}$ & 0 & 0 & 0 & 0 \\
\hline Pyroxasulfone (100) & $3^{\mathrm{b}}$ & $3^{\mathrm{b}}$ & 3 & 3 & 1 & 1 \\
\hline Pyroxasulfone (200) & $6^{\mathrm{bc}}$ & $7^{\mathrm{bc}}$ & 10 & 10 & 5 & 4 \\
\hline Sulfentrazone (210) & $6^{\mathrm{bc}}$ & $8^{\mathrm{bc}}$ & 9 & 7 & 4 & 3 \\
\hline Sulfentrazone (420) & $16^{\mathrm{cd}}$ & $23^{\mathrm{de}}$ & 25 & 23 & 12 & 7 \\
\hline Pyroxasulfone + Sulfentrazone $(100+210)$ & $11^{\mathrm{bcd}}$ & $10^{\mathrm{cd}}$ & 11 & 9 & 5 & 3 \\
\hline Pyroxasulfone + Sulfentrazone $(200+420)$ & $40^{\mathrm{d}}$ & $42^{\mathrm{e}}$ & 43 & 42 & 28 & 15 \\
\hline \multicolumn{7}{|l|}{ Interaction } \\
\hline $\mathrm{B} \times \mathrm{H}$ & NS & NS & ** & $*$ & $* *$ & ** \\
\hline \multicolumn{7}{|l|}{ Contrasts } \\
\hline Pyroxasulfone vs pyroxasulfone + sulfentrazone & 4 vs $22^{* *}$ & 4 vs $21^{* *}$ & 6 vs $22^{* *}$ & 6 vs $20^{* *}$ & 3 vs $12^{* *}$ & 2 vs $7^{* *}$ \\
\hline Sulfentrazone vs pyroxasulfone + sulfentrazone & 10 vs $22^{* *}$ & 13 vs 21 & 15 vs 22 & 13 vs $20^{*}$ & 7 vs $12^{*}$ & 4 vs 7 \\
\hline
\end{tabular}

${ }^{\mathrm{a}}$ Abbreviations: B, bean market class; H, herbicide treatment; NS, not significant at $\alpha=0.05$; PRE, pre-emergence; WAE, weeks after emergence. ${ }^{b}$ Means of main effects were only separated when the interaction was not significant. Significance of main effects, interactions and contrasts are denoted by ${ }^{*}$ for $P<0.05$ and ${ }^{* *}$ for $P<0.01$. ' Means followed by the same letter in each column are not significantly different according to Fisher's Protected LSD at $P<0.05$.

Table 2. Plant density, shoot dry weight, plant height, seed moisture content and yield of four market classes of dry bean treated PRE with sulfentrazone and pyroxasulfone, alone and in combination, near Ridgetown and Exeter, ON, Canada over two years $(2014,2015)$.

\begin{tabular}{|c|c|c|c|c|c|}
\hline Main Effects ${ }^{\mathrm{a}}$ & $\begin{array}{l}\text { Plant Density } \\
\# \mathrm{~m}^{-1} \text { row }\end{array}$ & $\begin{array}{l}\text { Shoot Dry } \\
\text { Weight } \\
\text { g. } \text { plant }^{-1}\end{array}$ & $\begin{array}{l}\text { Plant } \\
\text { Height } \\
\text { cm }\end{array}$ & $\begin{array}{c}\text { Seed Moisture } \\
\text { Content } \\
\%\end{array}$ & $\begin{array}{l}\text { Yield } \\
\text { T } \text { ha }^{-1}\end{array}$ \\
\hline Dry Bean Market Class & $*$ & ** & ** & $*$ & $* *$ \\
\hline Adzuki & 14 & 0.7 & 33.8 & 15.3 & 1.70 \\
\hline Kidney & 15 & 3.2 & 59.2 & 19.5 & 2.71 \\
\hline Small Red Mexican & 17 & 2.4 & 61.1 & 21.0 & 3.39 \\
\hline White & 17 & 1.5 & 52.1 & 19.5 & 3.60 \\
\hline Herbicide Treatment $\left(\mathrm{g} \cdot \mathrm{ai} \cdot \mathrm{ha}^{-1}\right.$ ) & $* *$ & ** & $* *$ & $* *$ & $* *$ \\
\hline Untreated & 17 & 2.2 & 56.4 & 18.4 & 3.16 \\
\hline Pyroxasulfone (100) & 17 & 2.0 & 54.8 & 18.0 & 2.93 \\
\hline Pyroxasulfone (200) & 17 & 1.7 & 51.7 & 18.7 & 2.78 \\
\hline Sulfentrazone (210) & 16 & 2.0 & 53.2 & 18.4 & 2.93 \\
\hline Sulfentrazone (420) & 15 & 1.6 & 47.7 & 19.3 & 2.57 \\
\hline Pyroxasulfone + Sulfentrazone $(100+210)$ & 16 & 1.9 & 53.2 & 18.5 & 3.05 \\
\hline Pyroxasulfone + Sulfentrazone $(200+420)$ & 13 & 1.3 & 43.4 & 19.9 & 2.33 \\
\hline
\end{tabular}




\section{Continued}

$$
\begin{gathered}
\text { Interaction } \\
\mathrm{B} \times \mathrm{H} \\
\text { Contrasts }
\end{gathered}
$$

Pyroxasulfone vs pyroxasulfone + sulfentrazone $\quad 17$ vs $15^{* *} \quad 1.9$ vs $1.6^{* *} \quad 53.3$ vs $48.3^{* *} \quad 18.3$ vs $19.2^{* *} \quad 2.85$ vs 2.68

Sulfentrazone vs pyroxasulfone + sulfentrazone $\quad 16$ vs $15 \quad 1.8$ vs $1.6 \quad 50.5$ vs $48.3 \quad 18.8$ vs $19.2 \quad 2.75$ vs 2.68

${ }^{\mathrm{a}}$ Significance of main effects, interactions and contrasts indicated by ${ }^{*}$ for $P<0.05$ and ${ }^{* *}$ for $P<0.01$. Means were not separated as the interaction was significant.

Table 3. Visible injury (\%) of four market classes of dry bean treated PRE with sulfentrazone and pyroxasulfone, alone and

\begin{tabular}{|c|c|c|c|c|c|c|c|c|c|c|c|c|}
\hline Herbicide Treatment $\left(\mathrm{g} \cdot \mathrm{ai} \cdot \mathrm{ha}^{-1}\right.$ ) & \multicolumn{3}{|c|}{ Adzuki Bean } & \multicolumn{3}{|c|}{ Kidney Bean } & \multicolumn{3}{|c|}{$\begin{array}{c}\text { Small Red } \\
\text { Mexican Bean }\end{array}$} & \multicolumn{3}{|c|}{ White Bean } \\
\hline Injury 3 WAE & \multicolumn{12}{|c|}{$\%$} \\
\hline Untreated & 0 & a & & 0 & a & & 0 & a & & 0 & a & \\
\hline Pyroxasulfone (100) & 5 & $\mathrm{~b}$ & Z & 3 & $\mathrm{~b}$ & $\mathrm{Z}$ & 2 & $\mathrm{~b}$ & Z & 3 & $\mathrm{~b}$ & $\mathrm{Z}$ \\
\hline Pyroxasulfone (200) & 22 & bc & Z & 5 & bc & $\mathrm{Z}$ & 7 & bc & $\mathrm{Z}$ & 10 & bc & $\mathrm{Z}$ \\
\hline Sulfentrazone (210) & 17 & bc & $\mathrm{Z}$ & 4 & $\mathrm{~b}$ & $\mathrm{Z}$ & 4 & bc & $\mathrm{Z}$ & 16 & cde & $\mathrm{Z}$ \\
\hline Sulfentrazone (420) & 51 & $\mathrm{~cd}$ & $\mathrm{Y}$ & 12 & bc & $\mathrm{Z}$ & 15 & $\mathrm{~cd}$ & $\mathrm{YZ}$ & 44 & de & $\mathrm{YZ}$ \\
\hline Pyroxasulfone + Sulfentrazone $(100+210)$ & 41 & $\mathrm{~cd}$ & $\mathrm{Y}$ & 6 & bc & $\mathrm{Z}$ & 5 & bc & $\mathrm{Z}$ & 12 & bcd & $\mathrm{YZ}$ \\
\hline Pyroxasulfone + Sulfentrazone $(200+420)$ & 86 & d & Z & 23 & c & $\mathrm{Z}$ & 29 & d & $\mathrm{Z}$ & 59 & e & $\mathrm{Z}$ \\
\hline Injury 4 WAE & \multicolumn{12}{|c|}{$\%$} \\
\hline Untreated & 0 & a & & 0 & a & & 0 & a & & 0 & a & \\
\hline Pyroxasulfone (100) & 5 & $\mathrm{~b}$ & $\mathrm{Z}$ & 3 & $\mathrm{~b}$ & $\mathrm{Z}$ & 2 & $\mathrm{~b}$ & $\mathrm{Z}$ & 2 & $\mathrm{~b}$ & $\mathrm{Z}$ \\
\hline Pyroxasulfone (200) & 23 & $\mathrm{~cd}$ & Z & 6 & bc & $\mathrm{Z}$ & 6 & bcd & Z & 9 & bc & $\mathrm{Z}$ \\
\hline Sulfentrazone (210) & 14 & bc & $\mathrm{Y}$ & 4 & $\mathrm{~b}$ & $\mathrm{YZ}$ & 2 & $\mathrm{~b}$ & $\mathrm{Z}$ & 12 & bc & $\mathrm{YZ}$ \\
\hline Sulfentrazone (420) & 49 & $\mathrm{~cd}$ & $\mathrm{Z}$ & 11 & bc & $\mathrm{Z}$ & 14 & $\mathrm{~cd}$ & $\mathrm{Z}$ & 37 & $\mathrm{~cd}$ & $\mathrm{Z}$ \\
\hline Pyroxasulfone + Sulfentrazone $(100+210)$ & 28 & $\mathrm{~cd}$ & $\mathrm{Y}$ & 5 & bc & $\mathrm{Z}$ & 4 & bc & $\mathrm{Z}$ & 11 & bc & $\mathrm{YZ}$ \\
\hline Pyroxasulfone + Sulfentrazone $(200+420)$ & 90 & d & $\mathrm{Z}$ & 21 & c & $\mathrm{Z}$ & 26 & d & $\mathrm{Z}$ & 62 & d & $\mathrm{Z}$ \\
\hline Injury 6 WAE & \multicolumn{12}{|c|}{$\%$} \\
\hline Untreated & 0 & a & & 0 & a & & 0 & a & & 0 & a & \\
\hline Pyroxasulfone (100) & 1 & $\mathrm{~b}$ & $\mathrm{Z}$ & 1 & $\mathrm{ab}$ & $\mathrm{Z}$ & 1 & $\mathrm{~b}$ & $\mathrm{Z}$ & 2 & $\mathrm{~b}$ & $\mathrm{Z}$ \\
\hline Pyroxasulfone (200) & 14 & c & $\mathrm{Z}$ & 3 & bc & $\mathrm{Z}$ & 3 & bc & $\mathrm{Z}$ & 4 & $\mathrm{~b}$ & $\mathrm{Z}$ \\
\hline Sulfentrazone (210) & 9 & bc & $\mathrm{Z}$ & 2 & bc & $\mathrm{Z}$ & 2 & bc & $\mathrm{Z}$ & 4 & $\mathrm{~b}$ & $\mathrm{Z}$ \\
\hline Sulfentrazone (420) & 39 & $\mathrm{~cd}$ & $\mathrm{Y}$ & 4 & bc & $\mathrm{Z}$ & 6 & bc & Z & 22 & $\mathrm{~b}$ & $\mathrm{YZ}$ \\
\hline Pyroxasulfone + Sulfentrazone $(100+210)$ & 17 & $\mathrm{~cd}$ & $\mathrm{Z}$ & 3 & bc & $\mathrm{Z}$ & 3 & bc & $\mathrm{Z}$ & 4 & $\mathrm{~b}$ & $\mathrm{Z}$ \\
\hline Pyroxasulfone + Sulfentrazone $(200+420)$ & 86 & $\mathrm{~d}$ & Y & 11 & c & $\mathrm{Z}$ & 12 & c & $\mathrm{Z}$ & 49 & c & $\mathrm{YZ}$ \\
\hline Injury 8 WAE & \multicolumn{12}{|c|}{$\%$} \\
\hline Untreated & 0 & a & & 0 & a & & 0 & a & & 0 & a & \\
\hline Pyroxasulfone (100) & 1 & $a b$ & Z & 2 & $\mathrm{~b}$ & $\mathrm{Z}$ & 1 & $\mathrm{ab}$ & $\mathrm{Z}$ & 1 & $a b$ & $\mathrm{Z}$ \\
\hline Pyroxasulfone (200) & 8 & bc & Z & 3 & $\mathrm{~b}$ & $\mathrm{Z}$ & 3 & $\mathrm{~b}$ & Z & 2 & $\mathrm{~b}$ & $\mathrm{Z}$ \\
\hline Sulfentrazone (210) & 6 & bc & $\mathrm{Z}$ & 2 & $\mathrm{~b}$ & $\mathrm{Z}$ & 1 & $\mathrm{~b}$ & $\mathrm{Z}$ & 2 & $\mathrm{~b}$ & $\mathrm{Z}$ \\
\hline Sulfentrazone (420) & 33 & $\mathrm{~cd}$ & $\mathrm{Y}$ & 4 & $\mathrm{~b}$ & $\mathrm{Z}$ & 2 & $\mathrm{~b}$ & Z & 9 & bc & $\mathrm{YZ}$ \\
\hline Pyroxasulfone + Sulfentrazone $(100+210)$ & 11 & c & $\mathrm{Z}$ & 2 & $\mathrm{~b}$ & $\mathrm{Z}$ & 1 & $\mathrm{~b}$ & $\mathrm{Z}$ & 2 & $\mathrm{~b}$ & $\mathrm{Z}$ \\
\hline Pyroxasulfone + Sulfentrazone $(200+420)$ & 67 & $\mathrm{~d}$ & $\mathrm{Y}$ & 9 & $\mathrm{~b}$ & $\mathrm{Z}$ & 4 & $\mathrm{~b}$ & $\mathrm{Z}$ & 20 & c & $\mathrm{YZ}$ \\
\hline
\end{tabular}
in combination, near Ridgetown and Exeter, ON, Canada over two years (2014, 2015). Means followed by the same letter in a row (Y-Z) or column (a-d) for each rating are not significantly different according to Fisher's Protected LSD at $P<0.05^{\mathrm{a}}$.

${ }^{\mathrm{a}}$ Abbreviations: PRE, pre-emergence; WAE, weeks after emergence. 
Mexican bean at $4 \mathrm{WAE}$, but injury was equivalent for both rates in the other market classes. Sulfentrazone (420 $\mathrm{g} \cdot \mathrm{ai} \cdot \mathrm{ha}^{-1}$ ) caused up to $51 \%, 12 \%, 15 \%$ and $44 \%$ injury in adzuki, kidney, small red Mexican and white bean, respectively.

Pyroxasulfone $\left(100 \mathrm{~g} \cdot a \mathrm{i} \cdot \mathrm{ha}^{-1}\right)+$ sulfentrazone $\left(210 \mathrm{~g} \cdot \mathrm{ai} \cdot \mathrm{ha}^{-1}\right)$ caused up to $41,6,5$, and $12 \%$ injury in adzuki, kidney, small red Mexican and white bean, respectively, and pyroxasulfone $\left(200 \mathrm{~g} \cdot \mathrm{a} \cdot \mathrm{h} \cdot \mathrm{ha}^{-1}\right)+$ sulfentrazone (420 $\mathrm{g} \cdot \mathrm{ai} \cdot \mathrm{ha}^{-1}$ ) caused up to $90,23,29$, and $62 \%$ injury, respectively (Table 3). In other studies examining PRE tank mixtures of a group 14 plus a group 15 herbicide on dry bean, dimethenamid-p/saflufenacil at the $1 \mathrm{X}$ rate caused up to $18 \%$ injury in dry bean, while the $2 \mathrm{X}$ rate caused up to $45 \%$ injury [20]. Fomesafen + s-metolachlor only caused up to $6.4 \%$ injury [21]. With the exception of white bean at $6 \mathrm{WAE}$, the level of injury produced by either herbicide combination was not greater than the injury produced by the respective rate of sulfentrazone on its own. Based on orthogonal contrasts, the addition of sulfentrazone to pyroxasulfone caused an increase in bean injury of $5 \%$ to $18 \%$ at $1,2,3,4,6$ and 8 WAE (Table 1). In contrast, the addition of pyroxasulfone to sulfentrazone caused an increase in bean injury of $5 \%$ to $12 \%$ at 1,4 and 6 WAE.

\subsection{Plant Density}

Kidney bean and small red Mexican bean densities were not reduced by any of the herbicide treatments (Table 4). Pyroxasulfone (100 and $\left.200 \mathrm{~g} \cdot \mathrm{ai} \cdot \mathrm{ha}^{-1}\right)$, sulfentrazone (210 and $\left.420 \mathrm{~g} \cdot \mathrm{ai} \cdot \mathrm{ha}{ }^{-1}\right)$, and pyroxasulfone (100 $\left.\mathrm{g} \cdot \mathrm{ai} \cdot \mathrm{ha}^{-1}\right)+$ sulfentrazone $\left(210 \mathrm{~g} \cdot \mathrm{ai} \cdot \mathrm{ha}^{-1}\right)$ did not reduce adzuki or white bean density relative to the control. In

Table 4. Plant density, shoot dry weight and plant height (expressed as percentages of the untreated control) of four market classes of dry bean treated PRE with sulfentrazone and pyroxasulfone, alone and in combination, near Ridgetown and Exeter, ON, Canada over two years $(2014,2015)$. Means followed by the same letter in a row (W-Z) or column (a-c) for each variable are not significantly different according to Fisher's Protected LSD at $P<0.05$.

\begin{tabular}{|c|c|c|c|c|c|c|c|c|c|c|c|c|}
\hline \multirow{2}{*}{$\begin{array}{c}\text { Herbicide Treatment }\left(\mathrm{g} \cdot \mathrm{ai} \cdot \mathrm{ha}^{-1} \text { ) }\right. \\
\text { Plant Density }\end{array}$} & \multicolumn{3}{|c|}{ Adzuki Bean } & \multicolumn{3}{|c|}{ Kidney Bean } & \multicolumn{3}{|c|}{$\begin{array}{c}\text { Small Red } \\
\text { Mexican Bean }\end{array}$} & \multicolumn{3}{|c|}{ White Bean } \\
\hline & \multicolumn{12}{|c|}{$\%$} \\
\hline Untreated & 100 & $\mathrm{a}$ & $\mathrm{Z}$ & 100 & $\mathrm{a}$ & $\mathrm{Z}$ & 100 & $\mathrm{a}$ & $\mathrm{Z}$ & 100 & $\mathrm{a}$ & $\mathrm{Z}$ \\
\hline Pyroxasulfone (100) & 100 & $\mathrm{a}$ & $\mathrm{Z}$ & 105 & $\mathrm{a}$ & $\mathrm{Z}$ & 98 & $\mathrm{a}$ & $\mathrm{Z}$ & 86 & $\mathrm{ab}$ & $\mathrm{Z}$ \\
\hline Pyroxasulfone (200) & 92 & $\mathrm{a}$ & $\mathrm{Z}$ & 104 & $\mathrm{a}$ & $\mathrm{Z}$ & 98 & $\mathrm{a}$ & $\mathrm{Z}$ & 97 & $\mathrm{a}$ & $\mathrm{Z}$ \\
\hline Sulfentrazone (210) & 84 & $\mathrm{ab}$ & $\mathrm{Z}$ & 102 & $\mathrm{a}$ & $\mathrm{Z}$ & 98 & $\mathrm{a}$ & $\mathrm{Z}$ & 98 & $\mathrm{a}$ & $\mathrm{Z}$ \\
\hline Sulfentrazone (420) & 71 & $\mathrm{ab}$ & $\mathrm{Z}$ & 98 & $\mathrm{a}$ & $\mathrm{Z}$ & 93 & $\mathrm{a}$ & $\mathrm{Z}$ & 84 & $\mathrm{ab}$ & $\mathrm{Z}$ \\
\hline Pyroxasulfone + Sulfentrazone $(100+210)$ & 78 & $\mathrm{ab}$ & $\mathrm{Z}$ & 100 & a & $\mathrm{Z}$ & 96 & $\mathrm{a}$ & $\mathrm{Z}$ & 98 & $\mathrm{a}$ & $\mathrm{Z}$ \\
\hline Pyroxasulfone + Sulfentrazone $(200+420)$ & 55 & $\mathrm{~b}$ & $\mathrm{Y}$ & 90 & $\mathrm{a}$ & $\mathrm{YZ}$ & 101 & $\mathrm{a}$ & $\mathrm{Z}$ & 67 & $\mathrm{~b}$ & $\mathrm{YZ}$ \\
\hline Shoot Dry Weight & \multicolumn{12}{|c|}{$\%$} \\
\hline Untreated & 100 & $\mathrm{a}$ & $\mathrm{X}$ & 100 & $\mathrm{a}$ & $\mathrm{Z}$ & 100 & $\mathrm{a}$ & $\mathrm{YZ}$ & 100 & $\mathrm{a}$ & $\mathrm{Y}$ \\
\hline Pyroxasulfone (100) & 95 & a & $\mathrm{X}$ & 93 & a & $\mathrm{Z}$ & 92 & $\mathrm{ab}$ & $\mathrm{YZ}$ & 83 & $\mathrm{ab}$ & $\mathrm{Y}$ \\
\hline Pyroxasulfone (200) & 79 & $\mathrm{a}$ & $\mathrm{X}$ & 85 & $\mathrm{a}$ & $\mathrm{Z}$ & 78 & $\mathrm{ab}$ & $\mathrm{YZ}$ & 67 & abc & $\mathrm{Y}$ \\
\hline Sulfentrazone (210) & 102 & $\mathrm{a}$ & $\mathrm{X}$ & 90 & $\mathrm{a}$ & $\mathrm{Z}$ & 90 & $\mathrm{ab}$ & $\mathrm{YZ}$ & 78 & $\mathrm{ab}$ & $\mathrm{Y}$ \\
\hline Sulfentrazone (420) & 67 & $\mathrm{ab}$ & $\mathrm{X}$ & 82 & $\mathrm{a}$ & $\mathrm{Z}$ & 70 & $\mathrm{ab}$ & $\mathrm{YZ}$ & 64 & bc & $\mathrm{Y}$ \\
\hline Pyroxasulfone + Sulfentrazone $(100+210)$ & 70 & $\mathrm{ab}$ & $\mathrm{X}$ & 99 & $\mathrm{a}$ & $\mathrm{Z}$ & 89 & $\mathrm{ab}$ & $\mathrm{Z}$ & 71 & $\mathrm{ab}$ & $\mathrm{Y}$ \\
\hline Pyroxasulfone + Sulfentrazone $(200+420)$ & 31 & $\mathrm{~b}$ & W & 86 & $\mathrm{a}$ & $\mathrm{Z}$ & 64 & $\mathrm{~b}$ & $\mathrm{Y}$ & 40 & $\mathrm{C}$ & $\mathrm{X}$ \\
\hline Plant Height & \multicolumn{12}{|c|}{$\%$} \\
\hline Untreated & 100 & $\mathrm{a}$ & $\mathrm{Y}$ & 100 & $\mathrm{a}$ & $\mathrm{Z}$ & 100 & $\mathrm{a}$ & $\mathrm{Z}$ & 100 & $\mathrm{a}$ & $\mathrm{Z}$ \\
\hline Pyroxasulfone (100) & 97 & $\mathrm{a}$ & $\mathrm{Y}$ & 97 & $\mathrm{a}$ & $\mathrm{Z}$ & 98 & $\mathrm{a}$ & $\mathrm{Z}$ & 96 & $\mathrm{a}$ & $\mathrm{YZ}$ \\
\hline Pyroxasulfone (200) & 84 & $\mathrm{ab}$ & $\mathrm{Y}$ & 96 & a & $\mathrm{Z}$ & 95 & $\mathrm{a}$ & $\mathrm{Z}$ & 90 & $\mathrm{ab}$ & $\mathrm{Z}$ \\
\hline Sulfentrazone (210) & 90 & $\mathrm{ab}$ & $\mathrm{Y}$ & 97 & $\mathrm{a}$ & $\mathrm{Z}$ & 98 & $\mathrm{a}$ & $\mathrm{Z}$ & 91 & $\mathrm{ab}$ & $\mathrm{YZ}$ \\
\hline Sulfentrazone (420) & 72 & bc & $\mathrm{Y}$ & 96 & a & $\mathrm{Z}$ & 91 & $\mathrm{a}$ & $\mathrm{Z}$ & 76 & bc & $\mathrm{YZ}$ \\
\hline Pyroxasulfone + Sulfentrazone $(100+210)$ & 87 & $\mathrm{ab}$ & $\mathrm{Y}$ & 98 & $\mathrm{a}$ & $\mathrm{Z}$ & 97 & $\mathrm{a}$ & $\mathrm{Z}$ & 92 & $\mathrm{ab}$ & $\mathrm{Z}$ \\
\hline Pyroxasulfone + Sulfentrazone $(200+420)$ & 54 & c & $\mathrm{Y}$ & 91 & $\mathrm{a}$ & $\mathrm{Z}$ & 87 & $\mathrm{a}$ & $\mathrm{Z}$ & 71 & c & $\mathrm{Z}$ \\
\hline
\end{tabular}


contrast, pyroxasulfone $\left(200 \mathrm{~g} \cdot \mathrm{ai} \cdot \mathrm{ha}^{-1}\right)+$ sulfentrazone $\left(420 \mathrm{~g} \cdot \mathrm{ai} \cdot \mathrm{ha}^{-1}\right)$ reduced adzuki and white bean density by $45 \%$ and 33\%, respectively. Dimethenamid-p/saflufenacil also reduced plant density by $49 \%$ [20]. Based on orthogonal contrasts, the addition of sulfentrazone to pyroxasulfone reduced dry bean density by 2 plants $\mathrm{m}^{-1}$ of row; however, there was no impact on dry bean density when pyroxasulfone was added to sulfentrazone (Table 2).

\subsection{Shoot Dry Weight}

Pyroxasulfone $\left(100 \mathrm{~g} \cdot \mathrm{ai} \cdot \mathrm{ha}^{-1}\right)$ and sulfentrazone $\left(210 \mathrm{~g} \cdot \mathrm{ai} \cdot \mathrm{ha}^{-1}\right)$ alone did not affect shoot dry weight for any of the dry bean market classes (Table 4). Pyroxasulfone (200 g.ai ha $\left.{ }^{-1}\right)$, sulfentrazone $\left(420 \mathrm{~g} \cdot \mathrm{ai}^{\mathrm{i}} \mathrm{ha}^{-1}\right)$, and pyroxasulfone $\left(100 \mathrm{~g} \cdot \mathrm{ai} \cdot \mathrm{ha}^{-1}\right)+$ sulfentrazone $\left(210 \mathrm{~g} \cdot \mathrm{ai}^{-h^{-1}} \mathrm{a}^{-1}\right.$ numerically reduced adzuki shoot dry weight by $21 \%$, $33 \%$, and $30 \%$, respectively, but not relative to the control. Pyroxasulfone $\left(200 \mathrm{~g} \cdot \mathrm{ai} \cdot \mathrm{ha}^{-1}\right)+$ sulfentrazone (420 $\mathrm{g} \cdot \mathrm{ai} \cdot \mathrm{ha}^{-1}$ ) reduced adzuki shoot dry weight by $69 \%$. Kidney bean shoot dry weight was not reduced by any of the herbicide treatments compared to the control. Pyroxasulfone $\left(200 \mathrm{~g} \cdot \mathrm{ai}^{\mathrm{h}} \mathrm{ha}^{-1}\right)+$ sulfentrazone $\left(420 \mathrm{~g} \cdot \mathrm{ai} \cdot \mathrm{ha}^{-1}\right)$ reduced small red Mexican bean shoot dry weight by $36 \%$. White bean shoot dry weight was not reduced compared to the control by pyroxasulfone $\left(100\right.$ and $\left.200 \mathrm{~g} \cdot \mathrm{ai} \cdot \mathrm{ha}^{-1}\right)$, sulfentrazone $\left(210 \mathrm{~g} \cdot \mathrm{ai}^{\mathrm{h}} \mathrm{ha}^{-1}\right)$, or pyroxasulfone $\left(100 \mathrm{~g} \cdot \mathrm{ai} \cdot \mathrm{ha}^{-1}\right)+$ sulfentrazone $\left(210 \mathrm{~g} \cdot \mathrm{ai} \cdot \mathrm{ha}^{-1}\right)$, but sulfentrazone $\left(420 \mathrm{~g} \cdot \mathrm{ai} \cdot \mathrm{ha}^{-1}\right)$ and pyroxasulfone $\left(200 \mathrm{~g} \cdot \mathrm{ai} \cdot \mathrm{ha}^{-1}\right)$ + sulfentrazone $\left(420 \mathrm{~g} \cdot \mathrm{ai}^{-h^{-1}}\right)^{-1}$ reduced shoot dry weight by $36 \%$ and $60 \%$, respectively. In other studies, pyroxasulfone $\left(209 \mathrm{~g} \cdot \mathrm{ai}^{\mathrm{h}} \cdot \mathrm{ha}^{-1}\right)$ reduced shoot dry weight by up to $30 \%$, and sulfentrazone $\left(280 \mathrm{~g} \cdot \mathrm{ai} \cdot \mathrm{ha} \mathrm{h}^{-1}\right)$ reduced shoot dry weight by up to $19 \%$ [18] [20]. Orthogonal contrasts indicate the addition of sulfentrazone to pyroxasulfone reduced bean shoot dry weight; however, there was no impact on shoot dry weight when pyroxasulfone was added to sulfentrazone (Table 2).

\subsection{Plant Height}

Taller plants are desirable for dry bean crops to reduce harvest losses. Pyroxasulfone and sulfentrazone, alone and in combination, did not reduce kidney or small red Mexican bean height. This contrasts other studies which found a 20\% decrease in pinto and small red Mexican bean height, and a 13\% decrease in kidney bean height when treated with $209 \mathrm{~g} \cdot \mathrm{ai} \cdot \mathrm{ha}^{-1}$ pyroxasulfone PRE [17] [21]. Pyroxasulfone (100 and $200 \mathrm{~g} \cdot \mathrm{ai} \cdot \mathrm{ha}^{-1}$ ), sulfentrazone $\left(210 \mathrm{~g} \cdot \mathrm{ai} \cdot \mathrm{ha}^{-1}\right)$, and pyroxasulfone $\left(100 \mathrm{~g} \cdot \mathrm{ai} \cdot \mathrm{ha}^{-1}\right)+$ sulfentrazone $\left(210 \mathrm{~g} \cdot \mathrm{ai} \cdot \mathrm{ha}^{-1}\right)$ also did not reduce adzuki or white bean height compared to the control. Sulfentrazone $\left(420 \mathrm{~g} \cdot \mathrm{ai}^{-\mathrm{ha}^{-1}}\right)$ reduced adzuki and white bean height by $28 \%$ and $24 \%$, respectively, and pyroxasulfone $\left(200 \mathrm{~g} \cdot a \mathrm{i} \cdot \mathrm{ha}^{-1}\right)+$ sulfentrazone $\left(420 \mathrm{~g} \cdot \mathrm{ai} \cdot \mathrm{ha}^{-1}\right)$ reduced adzuki and white bean height by $46 \%$ and $29 \%$, respectively. This is higher than in other studies, which found sulfentrazone $\left(420 \mathrm{~g} \cdot \mathrm{ai} \cdot \mathrm{ha}^{-1}\right)$ caused a $6 \%$ to $15 \%$ height reduction in black, brown, cranberry, kidney, otebo, pinto, white, and yellow eye beans [22]. Based on orthogonal contrasts, the addition of sulfentrazone to pyroxasulfone reduced bean height $5 \mathrm{~cm}$. However, there was no impact on bean height when pyroxasulfone was added to sulfentrazone (Table 2).

\subsection{Seed Moisture Content}

Elevated seed moisture content indicates a delay in crop maturity, which will cause increased drying costs. In this study, kidney, small red Mexican and white bean seed moisture content was not affected by any of the herbicide treatments compared to the control (Table 5). Pyroxasulfone $\left(200 \mathrm{~g} \cdot \mathrm{ai} \cdot \mathrm{ha}^{-1}\right)+$ sulfentrazone $\left(420 \mathrm{~g} \cdot \mathrm{ai} \cdot \mathrm{ha}^{-1}\right)$ increased adzuki bean seed moisture by $2.4 \%$. Given that the seed moisture content of adzuki bean treated with sulfentrazone (420 g $\cdot \mathrm{ai}^{\mathrm{h}} \mathrm{ha}^{-1}$ ) was not different from this combination, the delayed maturity can be attributed to the sulfentrazone rather than the pyroxasulfone. This is reflected by the orthogonal contrasts, which show the addition of sulfentrazone to pyroxasulfone increased bean moisture content (delayed maturity) at harvest by $0.9 \%$; however, there was no impact on bean maturity when pyroxasulfone was added to sulfentrazone (Table 2).

\subsection{Yield}

Kidney and small red Mexican bean yield were not affected by any of the herbicide treatments evaluated (Table 5). Pyroxasulfone and sulfentrazone applied on their own did not reduce white bean yield relative to the control, although yield was numerically reduced by $23 \%$ by sulfentrazone at $420 \mathrm{~g} \cdot \mathrm{ai} \cdot \mathrm{ha}^{-1}$. This is similar to other studies, where sulfentrazone $\left(420 \mathrm{~g} \cdot \mathrm{ai} \cdot \mathrm{ha}^{-1}\right)$ reduced black, brown, cranberry, kidney, otebo, pinto, white and yellow eye 
Table 5. Seed moisture content (\%) and yield (as a percent of the untreated control) of four market classes of dry bean treated PRE with sulfentrazone and pyroxasulfone, alone and in combination, near Ridgetown and Exeter, ON, Canada over two years (2014, 2015). Means followed by the same letter in a row (Y-Z) or column (a-c) are not significantly different according to Fisher's Protected LSD at $P<0.05$.

\begin{tabular}{|c|c|c|c|c|c|c|c|c|c|c|c|c|}
\hline Herbicide Treatment $\left(\mathrm{g} \cdot \mathrm{ai} \cdot \mathrm{ha}^{-1}\right)$ & \multicolumn{3}{|c|}{ Adzuki Bean } & \multicolumn{3}{|c|}{ Kidney Bean } & \multicolumn{3}{|c|}{$\begin{array}{c}\text { Small Red } \\
\text { Mexican Bean }\end{array}$} & \multicolumn{3}{|c|}{ White Bean } \\
\hline Seed Moisture Content & \multicolumn{12}{|c|}{$\%$} \\
\hline Untreated & 14.9 & a & Z & 19.7 & $\mathrm{a}$ & Z & 20.2 & a & Z & 19.1 & $\mathrm{a}$ & $\mathrm{Z}$ \\
\hline Pyroxasulfone (100) & 14.6 & a & Z & 19.1 & a & Z & 20.0 & a & Z & 18.9 & a & $\mathrm{Z}$ \\
\hline Pyroxasulfone (200) & 15.1 & a & $\mathrm{Z}$ & 20.2 & $\mathrm{a}$ & Z & 20.8 & a & $\mathrm{Z}$ & 19.1 & $\mathrm{a}$ & $\mathrm{Z}$ \\
\hline Sulfentrazone (210) & 14.9 & a & Z & 19.3 & $\mathrm{a}$ & $\mathrm{Z}$ & 20.8 & a & Z & 19.3 & a & $\mathrm{Z}$ \\
\hline Sulfentrazone (420) & 15.5 & $a b$ & Z & 19.7 & $\mathrm{a}$ & $\mathrm{YZ}$ & 22.4 & a & $\mathrm{Y}$ & 20.1 & $\mathrm{a}$ & $\mathrm{YZ}$ \\
\hline Pyroxasulfone + Sulfentrazone $(100+210)$ & 15.3 & a & $\mathrm{Z}$ & 19.1 & $\mathrm{a}$ & Z & 20.5 & a & $\mathrm{Z}$ & 19.4 & a & $\mathrm{Z}$ \\
\hline Pyroxasulfone + Sulfentrazone $(200+420)$ & 17.3 & $\mathrm{~b}$ & $\mathrm{Z}$ & 19.8 & $\mathrm{a}$ & $\mathrm{Z}$ & 22.1 & a & $\mathrm{Z}$ & 20.8 & a & $\mathrm{Z}$ \\
\hline Yield & \multicolumn{12}{|c|}{$\%$} \\
\hline Untreated & 100 & a & $\mathrm{Y}$ & 100 & $\mathrm{a}$ & $\mathrm{YZ}$ & 100 & a & $\mathrm{YZ}$ & 100 & $\mathrm{a}$ & $\mathrm{Z}$ \\
\hline Pyroxasulfone (100) & 95 & a & Z & 95 & $\mathrm{a}$ & $\mathrm{Z}$ & 92 & $\mathrm{a}$ & $\mathrm{Z}$ & 90 & $a b$ & $\mathrm{Z}$ \\
\hline Pyroxasulfone (200) & 83 & ab & $\mathrm{Y}$ & 89 & $\mathrm{a}$ & $\mathrm{YZ}$ & 89 & a & $\mathrm{YZ}$ & 89 & $a b$ & $\mathrm{Z}$ \\
\hline Sulfentrazone (210) & 82 & ab & $\mathrm{Y}$ & 98 & $\mathrm{a}$ & $\mathrm{YZ}$ & 99 & a & $\mathrm{Z}$ & 90 & $a b$ & $\mathrm{Z}$ \\
\hline Sulfentrazone (420) & 57 & bc & $\mathrm{Y}$ & 96 & $\mathrm{a}$ & $\mathrm{Z}$ & 93 & a & Z & 77 & $\mathrm{ab}$ & $\mathrm{Z}$ \\
\hline Pyroxasulfone + Sulfentrazone $(100+210)$ & 78 & ab & $\mathrm{Y}$ & 104 & $\mathrm{a}$ & YZ & 102 & a & $\mathrm{Z}$ & 98 & a & $\mathrm{Z}$ \\
\hline Pyroxasulfone + Sulfentrazone $(200+420)$ & 41 & c & $\mathrm{Y}$ & 87 & a & Z & 91 & $\mathrm{a}$ & $\mathrm{Z}$ & 72 & b & Z \\
\hline
\end{tabular}

bean yield by $15 \%, 0 \%, 11 \%, 0 \%, 3 \%, 0 \%, 17 \%$ and $0 \%$, respectively [22]. Pyroxasulfone $\left(100 \mathrm{~g} \cdot \mathrm{ai} \cdot \mathrm{ha}^{-1}\right)+\mathrm{sul}-$ fentrazone $\left(210 \mathrm{~g} \cdot \mathrm{ai}^{\mathrm{i}} \mathrm{ha}^{-1}\right)$ also did not reduce white bean yield relative to the control, but pyroxasulfone (200 $\left.\mathrm{g} \cdot \mathrm{ai} \cdot \mathrm{ha}^{-1}\right)+$ sulfentrazone $\left(420 \mathrm{~g} \cdot \mathrm{aiha}^{-1}\right)$ reduced yield by $28 \%$. Adzuki bean yield was the most affected of all the market classes evaluated. Sulfentrazone $\left(420 \mathrm{~g} \cdot a \cdot \mathrm{ha}^{-1}\right)$ reduced yield by $43 \%$ and pyroxasulfone (200 $\left.\mathrm{g} \cdot \mathrm{ai} \cdot \mathrm{ha} \mathrm{a}^{-1}\right)+$ sulfentrazone $\left(420 \mathrm{~g} \cdot \mathrm{ai} \cdot \mathrm{ha} \mathrm{a}^{-1}\right)$ reduced yield by $59 \%$. The yield reduction caused by pyroxasulfone + sulfentrazone in this study is lower than that caused by dimethenamid-p/saflufenacil, which caused up to $61 \%$ yield loss in white bean [20]. Based on orthogonal contrasts, there was no effect on bean yield when sulfentrazone was added to pyroxasulfone or when pyroxasulfone was added to sulfentrazone (Table 2).

\section{Conclusion}

In general, the level of injury caused by pyroxasulfone was lower and sulfentrazone was higher in this study than that reported in other studies, which might be a result of differential weather and soil conditions. Adzuki bean was the least tolerant to PRE mixtures of pyroxasulfone + sulfentrazone, with up to $90 \%$ injury and a 59\% yield loss. White bean was the second most sensitive, with a narrow margin of crop safety at the low rates, and kidney and small red Mexican bean had acceptable tolerance at the low rates. Based on the results of this study, pyroxasulfone $\left(100 \mathrm{~g} \cdot \mathrm{a} \cdot \mathrm{ha}^{-1}\right)+$ sulfentrazone $\left(210 \mathrm{~g} \cdot \mathrm{ai} \cdot \mathrm{ha}^{-1}\right)$ applied PRE is a safe herbicide combination for kidney and small red Mexican beans in Ontario.

\section{References}

[1] Kulasekera, K. (2015) Estimated Area, Yield, Production and Farm Value of Specified Field Crops, Ontario, 20112015. Ontario Ministry of Agriculture, Food and Rural Affairs.

http://www.omafra.gov.on.ca/english/stats/crops/estimate_new.htm\#metric 
[2] Malik, V.S., Swanton, C.J. and Michaels, T.E. (1993) Interaction of White Bean (Phaseolus vulgaris) Market Class, Row Spacing, and Seeding Density with Annual Weeds. Weed Science, 41, 62-68.

[3] Soltani, N., Nurse, R.E., Shropshire, C. and Sikkema, P.H. (2014) Weed Control in White Bean with Various Halosulfuron Tankmixes. Advances in Agriculture, 2014, Article ID: 391634. http://dx.doi.org/10.1155/2014/391634

[4] Soltani, N., Nurse, R.E., Shropshire, C. and Sikkema, P.H. (2014) Weed Control with Halosulfuron Applied Preplant Incorporated, Preemergence or Postemergence in White Bean. Agricultural Sciences, 5, 875-881. http://dx.doi.org/10.4236/as.2014.510094

[5] Heap, I. (2014) The International Survey of Herbicide Resistant Weeds. http://weedscience.org/summary/country.aspx

[6] Nandihalli, U.B. and Duke, S.O. (1993) The Porphyrin Pathway as a Herbicide Target Site. In: Duke, S.O., Menn, J.J. and Plimmer, J.R., Eds., Pest Control with Enhanced Environmental Safety, American Chemical Society Symposium Series 524, 62-78. http://dx.doi.org/10.1021/bk-1993-0524.ch005

[7] Shaner, D.L. (2014) Herbicide Handbook. 10th Edition, Weed Science Society of America, Lawrence, 513 p.

[8] Niekamp, J.W., Johnson, W.G. and Smeda, R.J. (1999) Broadleaf Weed Control with Sulfentrazone and Flumioxazin in No-Tillage Soybean (Glycine max). Weed Technology, 13, 233-238.

[9] Hancock, H.G. (2001) Sulfentrazone North American Field Performance: A Review in Efficacy in Selected Crops and Identification of Potential Partner Herbicides. FMC Corporation, 152 p.

[10] Niekamp, J.W. and Johnson, W.G. (2001) Weed Management with Sulfentrazone and Flumioxazin in No-Tillage Soyabean (Glycine max). Crop Protection, 20, 215-220. http://dx.doi.org/10.1016/S0261-2194(00)00129-0

[11] Anonymous (2012) Authority ${ }^{\circledR} 480$ Herbicide Product Label. FMC Corporation, 4 p.

[12] Anonymous (2006) KIH-485 Experimental Herbicide. Kumia Chemical Industry Co., Ltd., White Plains.

[13] Tanetani, Y. (2012) Action Mechanism of Isoxazoline-Type Herbicides. Journal of Pesticide Science, 37, $261-262$. http://dx.doi.org/10.1584/jpestics.J12-05

[14] Tanetani, Y., Fujioka, T., Kaku, K. and Shimizu, T. (2011) Studies on the Inhibition of Plant Very-Long-Chain Fatty Acid Elongase by a Novel Herbicide, Pyroxasulfone. Journal of Pesticide Science, 36, 221-228. http://dx.doi.org/10.1584/jpestics.G10-81

[15] Nurse, R.E., Sikkema, P.H. and Robinson, D.E. (2011) Weed Control and Sweet Maize (Zea mays L.) Yield as Affected by Pyroxasulfone Dose. Crop Protection, 30, 789-793. http://dx.doi.org/10.1016/j.cropro.2011.03.026

[16] Anonymous (2013) Fierce ${ }^{\circledR}$ Herbicide Product Label. Valent Publication 2013-FIE-0001. Valent USA, Corporation, Walnut Creek, 14 p.

[17] Sikkema, P.H., Robinson, D.E., Nurse, R.E. and Soltani, N. (2008) Pre-Emergence Herbicides for Potential Use in Pinto and Small Red Mexican Bean (Phaseolus vulgaris) Production. Crop Protection, 27, 124-129. http://dx.doi.org/10.1016/j.cropro.2007.04.017

[18] Soltani, N., Shropshire, C. and Sikkema, P.H. (2009) Response of Dry Bean to Preplant Incorporated and Preemergence Applications of Pyroxasulfone. Canadian Journal of Plant Science, 89, 993-997. http://dx.doi.org/10.4141/CJPS09058

[19] Soltani, N., Shropshire, C. and Sikkema, P.H. (2014) Response of Dry Bean to Sulfentrazone plus Imazethapyr. International Journal of Agronomy, 2014, Article ID: 287908. http://dx.doi.org/10.1155/2014/287908

[20] Soltani, N., Shropshire, C. and Sikkema, P.H. (2014) Sensitivity of Dry Bean to Dimethenamid-p, Saflufenacil and Dimethenamid-p/saflufenacil. American Journal of Plant Sciences, 5, 3288-3294. http://dx.doi.org/10.4236/ajps.2014.521343

[21] Sikkema, P.H., Shropshire, C. and Soltani, N. (2009) Response of Dry Bean to Pre-Plant Incorporated and Pre-Emergence Applications of s-Metolachlor and Fomesafen. Crop Protection, 28, 744-748. http://dx.doi.org/10.1016/j.cropro.2009.05.011

[22] Hekmat, S., Shropshire, C., Soltani, N. and Sikkema, P.H. (2007) Responses of Dry Beans (Phaseolus vulgaris L.) to Sulfentrazone. Crop Protection, 26, 525-529. http://dx.doi.org/10.1016/j.cropro.2006.05.002 